\title{
Adaptación de la escala Dragones de Inacción Barreras Psicológicas (DIPB) en población colombiana
}

\author{
Laura Sofía Alfonso Gutiérrez; Luis Enrique Prieto Patiño
}

Cómo citar este artículo:

Alfonso Gutierrez, L. S., \& Prieto Patiño, L. E. (2022). Adaptación de la escala Dragones de Inacción Barreras Psicológicas(DIPB) en población colombiana. Acta Colombiana de Psicología, 25(1), 183-202. https://www.doi.org/10.14718/ACP. 2022.25.1.12

Recibido, junio 30/2020; Concepto de evaluación, agosto 20/2021; Aceptado, noviembre 3/2021

\author{
Laura Sofía Alfonso Gutiérrez \\ ORCID: https://orcid.org/0000-0002-9579-7803 \\ Universidad de La Sabana, Chía, Colombia. \\ Luis Enrique Prieto Patiño ${ }^{1}$ \\ ORCID: https://orcid.org/0000-0002-3118-9892 \\ Universidad de La Sabana, Chía, Colombia.
}

Resumen

\begin{abstract}
El daño ambiental que se ha venido provocando a causa de la actividad humana es preocupante, razón por la cual se ha identificado como una de las posibles soluciones tener un comportamiento proambiental. Sin embargo, se ha visto que hay barreras psicológicas que impiden o dificultan esta conducta. Por este motivo, con el fin de tener un instrumento que permita evaluar estas barreras psicológicas en nuestro contexto, el objetivo de la presente investigación fue realizar la adaptación de la escala Dragons of Inaction Psychological Barriers (DIPB) en población colombiana. La muestra estuvo compuesta por 810 estudiantes universitarios de Bogotá y Chía, Colombia; 367 hombres y 443 mujeres con edades entre los 15 y los 48 años $(M=19.67, D E=2.414)$. En general, se realizó un análisis factorial exploratorio y un análisis factorial confirmatorio, con los cuales se comprobó la existencia de una estructura de cinco factores con 20 ítems; se realizaron análisis de reactivos y fiabilidad que demostraron que el instrumento tiene una alta consistencia interna; y se llevaron a cabo dos estudios de invarianza, con los que se demostró que la estructura factorial es invariante para todos los grupos de estudio. Además, se realizó la baremación del instrumento total. En conclusión, esta adaptación del DIPB presenta adecuada validez y fiabilidad, y permite guiar futuras investigaciones acerca de las barreras de inacción en el contexto latinoamericano, a la vez que aporta un insumo para realizar un primer diagnóstico de este constructo en Colombia.

Palabras clave: comportamiento proambiental, psicología ambiental, medio ambiente, análisis factorial, psicometría.
\end{abstract}

\section{Adaptation of the Dragons of Inaction Psychological Barriers (DIPB) scale in a Colombian population}

\begin{abstract}
The environmental damage that has been generated by human activity is a cause for concern, so pro-environmental behavior has been identified as one of the possible solutions. However, it has been seen that there are psychological barriers that prevent or hinder this behavior. For this reason, in order to have an instrument to evaluate these psychological barriers in our context, the objective of this research was to adapt the Dragons of Inaction Psychological Barriers (DIPB) scale to the
\end{abstract}

\footnotetext{
Facultad de Psicología, Universidad de La Sabana, Chía, Colombia. Campus del Puente del Común, Km 7, Autopista Norte de Bogotá, Chía, Colombia. luis.prieto@unisabana.edu.
} 
Colombian population. The sample was made up of 810 university students from Bogotá and Chía, Colombia; 367 males and 443 females between 15 and 48 years of age $(\mathrm{M}=19.67, \mathrm{SD}=2.414)$. In general, an exploratory factor analysis and a confirmatory factor analysis were carried out, which demonstrated the existence of a five-factor structure with 20 items. Item and reliability analyses were performed, which demonstrated that the instrument has a high internal consistency; and two invariance studies were carried out, which showed that the factor structure is invariant for all study groups. In addition, the total instrument was scored. In conclusion, this adaptation of the DIPB presents adequate validity and reliability, and allows guiding future research on inaction barriers in the Latin American context, while providing an input for a first diagnosis of this construct in Colombia.

Keywords: pro-environmental behavior, environmental psychology, environment, factor analysis, psychometrics.

\section{Introducción}

El medio ambiente está pasando por una situación preocupante que compromete a todas las regiones del planeta. En distintas zonas del mundo, diferentes fenómenos, como olas de calor, sequías, fuertes precipitaciones y ciclones tropicales, entre otros, son evidencia de la gravedad del problema ambiental (Intergovernmental Panel on Climate Change [IPCC], 2021). Desde hace décadas, distintos estudios han demostrado que el daño ambiental ha estado impulsado por la actividad humana, y que esta influencia ha contribuido a la aceleración del calentamiento global (IPCC, 2021). De hecho, como consecuencia de los daños causados se han identificado múltiples desafíos ambientales a nivel global, como lo son el cambio climático, la pérdida de la biodiversidad, la degradación de los ecosistemas, la contaminación del aire y del agua, y la sobreexplotación y agotamiento de recursos (United Nations Environment Programme [UNEP], 2016).

Ante esta problemática, un gran número de individuos tanto a nivel nacional como internacional han expuesto su preocupación (Herrera-Mendoza et al., 2016; Leiserowitz, 2006), y recientemente se ha identificado que, así como algunos de estos problemas se generaron debido a acciones humanas (IPCC, 2021), la solución se puede encontrar también en el cambio de comportamiento de las personas (Schultz \& Kaiser, 2012; Swim et al., 2009).

De este modo, se ha reconocido la importancia de tener un comportamiento proambiental con el fin de promover conductas orientadas a proteger el medio ambiente (Páramo, 2017). Incluso, se ha llegado a afirmar que el planeta no puede ser sostenible sin establecer patrones de conducta proambientales (Díaz-Marín \& Geiger, 2019). No obstante, aunque se espera que las personas tengan un comportamiento proambiental, diversas investigaciones han mostrado que este comportamiento no se lleva a cabo debido a barreras psicológicas que pueden impedirlo (Kollmuss \& Agyeman, 2002; Lorenzoni et al., 2007).

Específicamente, el comportamiento proambiental se refiere a aquellas conductas que buscan conscientemente minimizar el impacto negativo de las acciones propias en el mundo natural y construido (Kollmuss \& Agyeman, 2002). $\mathrm{Al}$ respecto, se ha querido establecer reglas que guíen el comportamiento proambiental, las cuales, según Páramo (2017), refieren a normas, actitudes, valores y creencias que, como comportamientos de tipo verbal en forma de reglas, intervienen en decisiones ambientales. No obstante, como afirman Lorenzoni et al. (2007), es poco probable que un individuo muestre este comportamiento si no tiene un compromiso personal hacia temas ambientales, lo que involucra la conciencia proambiental, es decir, un complejo de conocimiento ambiental, valores, actitudes e involucramiento emocional ligados tanto a factores internos - como la motivación - como a factores externos - como aspectos sociales y culturales-.

De esta manera, se puede establecer que el comportamiento ambiental depende de una amplia gama de factores (Páramo, 2017; Sandoval-Escobar et al., 2019; Stern, 2000), y entre ellos se pueden encontrar las denominadas barreras psicológicas, es decir, aspectos cognitivos, afectivos y conductuales que impiden, dificultan o conllevan a la inacción de comportamientos proambientales y que, por tanto, son obstáculos importantes que imposibilitan convertir la preocupación ambiental en acciones concretas (Gifford \& Chen, 2017; Lorenzoni et al., 2007).

Respecto al constructo de barreras psicológicas, Lorenzoni et al. (2007) realizaron tres estudios que sugieren que a pesar de que se encuentre una amplia conciencia y preocupación 
por el cambio climático, esto por lo general no se traduce en compromiso personal, y al respecto identificaron una serie de barreras que impiden las acciones proambientales. Asimismo, en un contexto más cercano, Páramo et al. (2015) mostraron que América Latina enfrenta múltiples problemas ambientales, que las personas se muestran pesimistas acerca del estado actual y el futuro del medio ambiente, y que ellas mismas señalan la responsabilidad que tienen los agentes sociales externos sobre esta situación; sin embargo, también es importante recordar que el compromiso individual tiene implicaciones grandes en cuanto al comportamiento proambiental y, por ende, una gran influencia sobre el cambio que se puede causar en el medio ambiente (Páramo et al., 2015).

Así, vemos que, a pesar de que se reconoce el problema ambiental, y a pesar de que pueda existir preocupación ante este, las barreras psicológicas pueden evitar o dificultar el desarrollo de una conducta proambiental (Gifford \& Chen, 2017; Lorenzoni et al., 2007). Al hacer una revisión de la literatura sobre el tema, diversos autores han identificado un gran número de barreras de inacción (véase Tabla 1). En general, estas barreras recopilan aspectos en común, incluidos factores demográficos — como la edad—, factores externos - como la infraestructura, aspectos económicos, sociales y culturales-, y factores internos - como motivación, conocimiento ambiental, valores, actitudes, advertencia ambiental, compromiso emocional, locus de control, responsabilidad y prioridades - (Lorenzoni et al., 2007).

Ahora bien, teniendo como modelo base de esta investigación la teoría de Gifford (2011), se hace relevante explicar las 29 barreras psicológicas que el autor identifica, con la intención de entender la brecha existente entre actitud y comportamiento. El autor llama a estas barreras "dragones de inacción", y las clasifica en siete categorías (véase Gifford, 2011):

1. Cognición limitada: hace referencia al desconocimiento de cómo ayudar, resolver o implementar un cambio de comportamiento proambiental. Esta incluye, específicamente, el cerebro antiguo, la ignorancia, el entumecimiento ambiental, la incertidumbre, el descuento crítico, el sesgo de optimismo y el control conductual percibido o autoeficacia.

2. Ideologias: incluyen creencias o cosmovisiones, como la creencia de poderes suprahumanos, la salvación tecnológica y la justificación del sistema.

3. Comparación con otras personas: se reconocen las presiones ambientales como dificultad para un comportamiento proambiental. Aquí se incluye la

Tabla 1.

Barreras de inacción identificadas en la literatura

\begin{tabular}{ll}
\hline \multicolumn{1}{c}{ Autor } & \multicolumn{1}{c}{ Barreras de inacción } \\
\hline Blacke (1999) & Individualidad, responsabilidad y sentido práctico. \\
& Limitaciones cognitivas que dificultan la advertencia ambiental debido a la no \\
Preuss (1991) & $\begin{array}{l}\text { inmediatez de muchos problemas ecológicos, la destrucción ecológica lenta y gra- } \\
\text { dual, y la complejidad para entender los problemas ambientales. }\end{array}$ \\
& Comodidad, culpar al colectivo, creer en soluciones tecnológicas y dudar del com- \\
Stoll-Kleemann et al. (2001) & promiso del gobierno con la acción.
\end{tabular}

Kollmuss \& Agyeman (2002)

Carrus et al. (2008)

Dietz et al. (2009)

Lorenzoni et al. (2007)

Geiger et al. (2017)
Factores demográficos, y factores internos y externos.

Hábitos.

Falta de cosmovisiones y creencias ambientales.

Categoría individual: falta de conocimiento, incertidumbre y escepticismo, desconfianza en las fuentes de información, responsabilidad externa y culpa, el cambio climático es una amenaza lejana, otras cosas son más importantes y renuencia a cambiar los estilos de vida.

Categoría social: falta de acción política, falta de acción por parte de las empresas y la industria, preocuparse por el efecto de piloto libre, normas sociales y expectativas, y falta de iniciativas habilitadoras.

La falta de comprensión de las causas y del acuerdo científico sobre el cambio climático, la experiencia limitada con la amenaza, las visiones del mundo y las normas sociales. 
comparación social, las redes y normas sociales, y la inequidad percibida.

4. Costos hundidos: se refieren a las formas de inversión que dificultan el cambio, incluyendo inversiones financieras, impulso de comportamiento y valores, metas y aspiraciones conflictivas.

5. Discrepancia: incluye la inadecuación percibida de programas ambientales, la desconfianza de las autoridades, la resistencia reactiva y la negación de problemas.

6. Riesgo percibido: incluye el riesgo en cuanto al aspecto funcional, físico, financiero, social, psicológico y temporal.

7. Comportamiento limitado: el cual se puede dar debido al "tokenismo", que se refiere a la creencia general sobre los cambios propios de comportamiento y de cómo estos pueden ser suficientes para ayudar al ambiente y, por ello, no es necesario hacer mayor esfuerzo. Este factor incluye la opinión acerca de que hay agentes externos - como las industrias - que están causando un daño ambiental mayor, y que, por lo tanto, son esos agentes quienes deberían cambiar su comportamiento y no cada persona. Esta barrera también abarca la creencia acerca de que hay variables externas que deberían facilitar el cambio de comportamiento; un ejemplo de esto serían las disposiciones de los gobiernos y el efecto rebote (Lacroix et al., 2019).

Estas Barreras Psicológicas de Dragones de Inacción (DIPB) han sido evaluadas principalmente en relación con dilemas ambientales (Chen \& Gifford, 2015), y con varios dominios ambientales como la intención de elección de alimentos climáticamente positivos (Gifford \& Chen, 2017) y la conservación de energía del hogar (Lacroix \& Gifford, 2018). Sin embargo, se reconoce que estos estudios tienen algunas limitaciones, como implicar solo dominios ambientales específicos, no involucrar las siete categorías de los dragones de inacción y redactar los ítems de manera distinta en cada estudio (Lacroix et al., 2019). Asimismo, Lacroix et al. (2019) manifiestan como debilidades de estos estudios que la medición de las barreras fue de un solo elemento, $\mathrm{y}$ que, por consiguiente, tienen menor confiabilidad que las medidas con múltiples elementos (DeVellis, 1991; Wegener $\&$ Fabrigar, 2008). Incluso, se informa que los estudios no probaron la invarianza de la medición de la escala, lo cual es necesario para confirmar que los elementos y factores signifiquen lo mismo para distintos sujetos, con el fin de que las diferencias de grupos no se le atribuyan a las de medición, y así poder comparar adecuadamente las barreras con otros estudios (Cheung \& Rensvold, 2009; Milfont \& Fischer, 2010; Vandenberg \& Lance, 2000).

Por estos motivos, Lacroix et al. (2019) diseñaron un instrumento válido, confiable y definitivo para identificar las barreras psicológicas del comportamiento proambiental, llamado Dragons of Inaction Psychological Barriers (DIPB). En su investigación original, los autores llevaron a cabo tres estudios: en el primero, el instrumento pasó de tener 65 ítems a 24, y se determinó un modelo de cinco factores a través de un análisis factorial exploratorio; en el segundo, mediante un análisis factorial confirmatorio, se eliminaron dos ítems más - aunque se sugiere utilizarlos si el gobierno toma un papel importante en cuestiones ambientales-, se compararon los modelos de uno, cinco y siete factores, y se estableció que el modelo de cinco factores es el más viable; y en el tercero, se validó la escala en múltiples dominios ambientales por medio del análisis factorial confirmatorio multigrupo.

Teniendo en cuenta que el presente estudio se centra en la escala Dragons of Inaction Psychological Barriers (DIPB), es necesario identificar y definir los factores que para Lacroix et al. (2019) evalúa el instrumento. Según estos autores, los cinco factores que evalúa la escala son:

1. El cambio innecesario, que implica la negación de los problemas ambientales y de la necesidad de actuar frente a estos.

2. Las metas y aspiraciones conflictivas, en donde se abarca el tema de los objetivos que entran en conflicto, ya sea por la limitación del tiempo, por inversiones pasadas, o debido a la dificultad para cambiar los hábitos.

3. Las relaciones interpersonales, que cubre temas de desaprobación o crítica social.

4. La falta de conocimiento, que refiere a la cognición limitada, es decir, la ignorancia en el sentido en que la persona desconoce cómo cambiar su comportamiento en pro del ambiente.

5. Y el tokenismo, es decir, la creencia general acerca de que los cambios de comportamiento personales son suficientes y no es necesario hacer mayor esfuerzo; 
así como la creencia acerca de que agentes externos deberían facilitar el cambio de comportamiento.

En pocas palabras, las distintas investigaciones sobre las barreras psicológicas han mostrado la importancia de su estudio y medición, ya que pueden ser un medio para fomentar un cambio de comportamiento que podría provocar efectos que ayudarían a la desaceleración del cambio climático (Gifford \& Chen, 2017). En este sentido, se identificó la necesidad de contar con un instrumento válido y adecuado para medir las barreras psicológicas en español, y así tener un primer diagnóstico en el contexto colombiano. Asimismo, debido a la rigurosidad con la que se realizó el instrumento de Lacroix et al. (2019), y su buen funcionamiento para medir las barreras psicológicas, el presente estudio tuvo como objetivo realizar la adaptación de la escala Dragones de Inacción Barreras Psicológicas (DIPB) en Colombia.

Con el fin de adaptar este instrumento al contexto colombiano, en esta investigación se utilizaron las directrices descritas por Muñiz et al. (2013), elaboradas por la Comisión Internacional de Tests (ITC) para la adecuada adaptación de instrumentos de medición. Específicamente, primero se tuvieron en cuenta las directrices previas, es decir, la obtención del permiso legal del propietario del instrumento y la evaluación de la importancia del constructo en el contexto. Posteriormente, según las directrices de desarrollo, se garantizó una adaptación adecuada del instrumento en cuanto a diferencias culturales y lingüísticas -mediante un proceso de back translation -, teniendo en cuenta las instrucciones, los ítems, el formato y la forma de aplicación (Muñiz et al., 2013). Luego se procedió con las directrices de confirmación, con lo cual se seleccionó una muestra teniendo en cuenta características y tamaño apropiado, y se revisó la fiabilidad y validez de la prueba. Además, se tuvieron en cuenta las directrices de aplicación, pues se dispuso de los elementos necesarios para reducir diferencias contextuales y se estableció bajo cuáles condiciones se debe realizar la aplicación de la prueba (Muñiz et al., 2013). Después, según las directrices sobre puntuación e interpretación, se determinó el grado de comparación que pueden tener las puntuaciones. Y, por último, según las directrices sobre la documentación, se elaboró la información pertinente para corroborar la adecuada adaptación y la respectiva instrucción para el buen uso del instrumento (Muñiz et al., 2013).

\section{Método}

\section{Tipo de estudio}

Para la presente investigación se utilizó un diseño de tipo instrumental, mediante el cual fue posible analizar las propiedades psicométricas del instrumento (Ato et al., 2013), según los parámetros de adaptación de Muñiz et al. (2013).

\section{Participantes}

Se realizó un muestreo no probabilístico por conveniencia, mediante el cual se seleccionaron sujetos que fueron accesibles y que dieron su aprobación para ser parte del estudio (Otzen \& Manterola, 2017). Así, se obtuvo una muestra conformada por 810 estudiantes universitarios, 367 hombres $(45.31 \%$, ) y 443 mujeres $(54.69 \%)$, cuya edad oscilaba entre los 15 y los 48 años $(M=19.67, D E=2.41)$ - el promedio de edad para los hombres fue de 19.74 años $(D E=2.62)$, y para las mujeres fue de 19.61 años $(D E=2.21)$ — Con el fin de dar mayor representatividad de la muestra a la población colombiana, se seleccionó participantes de distintas carreras universitarias y semestres, y de varios niveles socioeconómicos (véase Tabla 2).

Tabla 2.

Características sociodemográficas de los participantes

\begin{tabular}{clcc}
\hline Variables & \multicolumn{1}{c}{ Nombre } & $n$ & $\%$ \\
\hline \multirow{5}{*}{ Carrera } & Ingeniería & 238 & 29.4 \\
& Psicología & 185 & 22.8 \\
& Ciencias económicas y & 153 & 18.9 \\
& administrativas & 72 & 8.9 \\
& Comunicación & 44 & 5.4 \\
& Derecho y ciencias políticas & 22 & 2.7 \\
& Educación y filosofía & 16 & 2 \\
& Gastronomía & 80 & 9.9 \\
& Enfermería, fisioterapia y & & \\
& medicina & 415 & 51.4 \\
& Entre primero y cuarto & 334 & 41.2 \\
Semestre & Entre quinto y octavo & 60 & 7.4 \\
& Entre noveno y decimocuarto & & \\
& Bajo & 24 & 3 \\
Nivel socio- & Medio & 601 & 74.2 \\
económico & Alto & 185 & 22.8 \\
\hline
\end{tabular}

La muestra total se dividió de la siguiente manera: para el análisis factorial exploratorio se utilizó el $30.9 \%$ de la muestra, es decir, un $n=250-97$ hombres $(38.8 \%)$ y 
153 mujeres (61.2 \%) -; luego, se llevó a cabo el análisis factorial confirmatorio, en el cual se utilizó el $69.1 \%$ de la muestra, es decir, $n=560-270$ hombres $(48.21 \%)$ y 290 mujeres (51.79\%) —; posteriormente, para el análisis de invarianza por tamaño se utilizó un $n=460$, y para el análisis de invarianza por sexo un $n=560$-para el análisis de reactivos y de fiabilidad del instrumento, la muestra fue la misma que en el análisis factorial confirmatorio, es decir, $n=560-; y$, por último, para la baremación del instrumento se utilizó un $n=535-252$ hombres (47\%) y 283 mujeres $(53 \%)$ - Se excluyeron personas mayores a 50 años.

\section{Instrumento}

Se utilizó la escala Dragons of Inaction Psychological Barriers (DIPB), diseñada originalmente por Lacroix et al. en Canadá, publicada en 2019. Como se mencionó anteriormente, esta escala se compone por lo general de 22 ítems, sin embargo, en este estudio se utilizaron 24 ítems, debido a que los autores recomiendan tenerlos en cuenta en algunos contextos. Para cada uno de estos ítems se tiene una escala de respuesta tipo Likert de siete puntos — de 1 = "muy en desacuerdo" a 7 = "muy de acuerdo"- En el diseño de esta escala, se realizaron estudios factoriales confirmatorios con los cuales se encontró un modelo de cinco factores con adecuados índices de bondad de ajuste $\left(x^{2}=555.71\right.$; $x^{2} / d f=2.79 ; \mathrm{CFI}=0.84 ;$ RMSEA $=0.078$, IC $90 \%[.07, .09]$; SRMR $=0.08 ; \mathrm{BIC}=22087.88$ ).

En este estudio se utilizó la versión de la escala de cinco factores, que son: (a) cambio innecesario $(\alpha=.85)$, compuesto por los ítems 1, 2, 3, 4 y 5; (b) metas y aspiraciones conflictivas $(\alpha=.82$ ), compuesto por los ítems $6,7,8,9$ y 10 ; (c) relaciones interpersonales $(\alpha=.80)$, compuesto por los ítems 11, 12, 13 y 14; (d) falta de conocimiento $(\alpha=.73)$, compuesto por los ítems 15,16 y 17 ; y (e) tokenismo $(\alpha=.77)$, compuesto por los ítems 18, 19, 20, 21 y 22.

\section{Procedimiento}

La investigación se llevó a cabo en nueve fases, siguiendo las recomendaciones de Muñiz et al. (2013):

- Fase 1. Traducción de la escala mediante el proceso de back translation, con la colaboración de cuatro traductores para garantizar la equivalencia lingüística y cultural.

- Fase 2. Validación por jueces, quienes evaluaron la validez del contenido teniendo en cuenta la suficiencia de los factores y la coherencia, relevancia y claridad de cada uno de los ítems. Se realizaron los ajustes pertinentes planteados por los jueces expertos.

- Fase 3. Aplicación experimental a 810 estudiantes universitarios de ambos sexos.

- Fase 4. Construcción y depuración de la base de datos en Excel con los datos obtenidos.

- Fase 5. Análisis factorial exploratorio, estudiando la estructura de los ítems, desde un factor hasta siete factores — dos más por encima del planteamiento teórico-.

- Fase 6. Análisis factorial confirmatorio con la estructura factorial encontrada de cinco factores.

- Fase 7. Estudio de la invarianza del modelo con la variable sexo.

- Fase 8. Análisis de reactivos y estudio de la fiabilidad del instrumento.

- Fase 9. Baremación del instrumento.

\section{Consideraciones éticas}

En primera instancia, se obtuvo el permiso de los autores de la prueba original para realizar la investigación, y, posteriormente, se diseñó el consentimiento informado, el cual fue presentado a los participantes - $\mathrm{O}$ a sus tutores en el caso de los participantes menores de edad-e incluyó los estándares mínimos de este tipo de documentos — cada participante debía incluir su firma de aprobación-. La información recolectada fue archivada manteniendo la confidencialidad.

La investigación fue avalada por el comité de investigaciones de la Facultad de Psicología de la Universidad de la Sabana para su realización, y es considerada un estudio sin riesgo, dada la naturaleza de las variables (Resolución 8430 de 1993). En concordancia con la Ley 1090 de 2006, se garantizó el respeto, la dignidad, el bienestar y los derechos de cada uno de los participantes durante toda la investigación.

\section{Análisis de datos}

Se realizó el análisis factorial exploratorio con los programas estadísticos Factor Analysis, versión 10.10.03, y JASP, versión 0.12.1; el análisis factorial confirmatorio con JASP, versión 0.12.1, y EQS, versión 6.1; el análisis de invarianza con EQS, versión 6.1; el análisis de reactivos y 
el análisis de fiabilidad del instrumento con JASP, versión 0.12.1; y la baremación del instrumento a partir de las puntuaciones típicas (puntuación T), la puntuación normalizada (puntuación Z) y la puntuación centil.

\section{Resultados}

A continuación se presentan los resultados de la traducción del instrumento y de la validación por jueces, seguidos de los hallazgos respecto al análisis factorial exploratorio y el análisis factorial confirmatorio, así como de los resultados del análisis de reactivos y el análisis de fiabilidad. Después se muestran los resultados del análisis de invarianza por tamaño y por sexo, y, finalmente, se presenta la baremación del instrumento.

\section{Back translation y validación por jueces}

En la presente investigación, el primer paso que se llevó a cabo fue el proceso de back translation, en el cual, inicialmente, la escala fue traducida al español por dos traductores certificados; luego, la versión en español se tradujo nuevamente al inglés por otros dos traductores certificados; y, posteriormente, se tomaron los mejores ítems de cada versión traducida y se conformó una versión general del instrumento en español. Esta versión fue revisada por un último traductor, y, tras incluir sus aportes, se realizó el análisis de validez de contenido mediante la validación por jueces, para la cual se sugiere tener un mínimo de cinco jueces (Escobar-Pérez \& Cuervo-Martínez, 2008). Específicamente, se contó con la participación de cinco jueces expertos en psicometría, investigación y barreras psicológicas.

En este punto, se analizaron los criterios de coherencia, relevancia y claridad para cada uno de los ítems, así como el criterio de suficiencia para cada factor; todos estos criterios fueron calificados de 1 a 4 . Se utilizó el formato de validación por jueces creado por Escobar-Pérez y CuervoMartínez (2008) para este procedimiento.

Como resultado, en el criterio de suficiencia, los tres primeros factores obtuvieron una calificación de 3.8, y los dos últimos factores fueron calificados con 3.6; en el criterio de coherencia, 16 ítems obtuvieron una puntuación por encima de 3.8 - lo que equivale al $66.66 \%$-, seis ítems obtuvieron una calificación entre 3.4 y 3.6 -que equivale al $24.96 \%$ - y dos ítems puntuaron 3 -lo que equivale al $8.3 \%$ -

En el criterio de relevancia, ocho ítems estuvieron por encima de 3.8 - lo que equivale al $33.32 \%$ - 11 ítems estuvieron entre 3.4 y 3.6 - lo que equivale al $45.83 \%$-, y cinco ítems puntuaron entre 3 y 3.2 -lo que equivale al $20.83 \%$ - Finalmente, en el criterio de claridad, 10 ítems estuvieron por encima de 3.8 -lo que equivale al $41.63 \%$ 11 ítems obtuvieron entre 3.4 y 3.6 -lo que equivale al $45.83 \%$ - y tres ítems obtuvieron una calificación de 3.2 - lo que equivale al $12.5 \%$ - De los 24 ítems, se observa que todos obtuvieron índices por encima del promedio esperado - de 3 puntos - , por tanto, se decidió dejar la versión completa, y no se modificó ni se eliminó ningún ítem.

\section{Análisis factorial exploratorio}

Para el análisis factorial exploratorio se utilizó el 30.9\% de la muestra total $(n=810)$, es decir, 250 sujetos. Primero, se determinó la normalidad univariada mediante la asimetría y curtosis de las variables, en este caso, los ítems. Como resultado, todos los valores estuvieron dentro de lo esperado - asimetría $<2$ y curtosis $<7$ - (Abad et al., 2011). Posteriormente, se analizó la normalidad multivariada con la prueba de Shapiro-Wilk como segundo requisito de los datos, con lo cual se obtuvo un valor de 0.715 , con un $p<.001$, lo que indica que se puede rechazar la hipótesis nula de normalidad multivariada. Así, debido a que no se cumplen los dos requisitos - tanto de normalidad univariada como multivariada - , se utilizó el método de estimación de mínimos residuales - encontrado en otros programas estadísticos como mínimos cuadrados no ponderados-, el cual no tiene en cuenta supuestos de distribución (LloretSegura et al., 2014).

Posterior a esto, para el estudio de la estructura factorial se utilizó el método de mínimos residuales, con rotación promax y normalización kaiser. Específicamente, se estudió la estructura factorial con uno, dos, tres, cuatro, cinco, seis y siete factores, y se encontró que la única estructura satisfactoria fue la de cinco factores. Durante el proceso, se determinó que algunos ítems debían ser removidos, debido a que no saturaban en ningún factor -ítem 2 y 6 - o se situaban solo dos ítems en un mismo factor -ítem 23 y $24-$, lo cual es inaceptable para establecer un factor (Raubenheimer, 2004). De este modo, como resultado se obtuvo una estructura factorial de cinco factores. 
Los reactivos eliminados fueron el ítem 2 - "Los humanos son impotentes cuando se trata de salvar la Tierra, por los que no hay necesidad de cambiar"-, el ítem 6 - "Hacer este cambio interferiría demasiado con mis otras metas en la vida"-, el ítem 23 - "El gobierno debería facilitarme este cambio, si realmente tiene en mente el mejor interés del medio ambiente" - y y el ítem 24 - "Es responsabilidad del gobierno regular este cambio"-.

En la Tabla 3 se presentan los índices que se examinaron para precisar el modelo de cinco factores. Específicamente, los índices fueron la medida de adecuación muestral de Kaiser-Meyer-Olkin (KMO), cuyo valor esperado es $\geq 0.80$; la relación $\chi^{2} / d f$, la cual sugiere que, al tener un valor menor a 3, presenta un buen ajuste; el RMSEA (Root Mean-Square Error of Approximation), cuyo valor esperado es $<0.08$; $\mathrm{y}$, por último, el TLI (Tucker-Lewis index), que sugiere obtener un valor $\geq 0.95$ (Lloret-Segura et al., 2014; Ruiz et al., 2010). Como se puede observar, los valores obtenidos indican un buen ajuste.

Por otra parte, en la Tabla 4 se encuentra la estructura factorial de cinco factores claramente constituidos, ya que cada uno incluye tres o más variables (Raubenheimer, 2004). Adicionalmente, respecto a la varianza explicada, el primer factor explica el $32.905 \%$, el segundo el $10.288 \%$, el tercero el $5.236 \%$, el cuarto el $3.567 \%$, y el quinto el $2.634 \%$, para un total de $54.6 \%$ de la varianza total explicada, lo cual se considera muy adecuado, ya que se requiere que se explique un mínimo de $40 \%$ de la varianza (Lloret-Segura et al., 2014).

Como se puede observar, todos los ítems tuvieron una saturación $\geq 0.40$ en cada uno de los factores a los que pertenecen, debido a que Stevens (2002) recomienda interpretar solamente las saturaciones $>0.40$. Asimismo, se considera que los reactivos deberían tener una comunalidad $\geq 0.40$, y, si se encuentra un valor menor a este, el investigador decide si excluir o no el ítem (Costello \& Osborne, 2005). En este caso, todos los ítems presentaron una comunalidad $>0.40$, a excepción de los ítems 1 y 18 , sin embargo, se decidió dejarlos, ya que presentan una saturación adecuada en el factor.
Finalmente, tal como se observa en la Tabla 4, la estructura factorial del DIPB presentó cinco factores: el primero, relaciones interpersonales, conformado por los ítems 11, 12, 13 y 14; el segundo, cambio innecesario, compuesto por los ítems 1, 3, 4 y 5; el tercero, tokenismo, conformado por los ítems 18, 19, 20, 21 y 22; el cuarto, falta de conocimiento, compuesto por los ítems 15, 16 y 17; y el quinto, metas y aspiraciones conflictivas, conformado por los ítems 7, 8, 9 y 10 , de acuerdo a la numeración original del instrumento. La estructura obtenida se contrastó a través de un análisis factorial confirmatorio.

\section{Análisis factorial confirmatorio}

El modelo de cinco factores encontrado en el análisis factorial exploratorio se puso a prueba en el análisis factorial confirmatorio, para el cual el tamaño de la muestra fue de 560 , es decir, el $69.1 \%$ de la muestra total $(n=810)$. En primera instancia, como recomiendan Martínez et al. (2014), se identificaron 100 datos extremos (outliers) a través del análisis de distancia extrema - mediante la prueba de Mahalanobis al cuadrado $\left(D^{2}\right)$ - con el fin de realizar el análisis factorial confirmatorio con y sin estos datos. La eliminación de estos datos también se realiza con el propósito de calcular la normalidad multivariada (Rigo \& Donolo, 2018).

Así, se pudo determinar que los resultados del análisis no mostraban una gran diferencia eliminando los outliers, y en ambos casos no se encontró normalidad multivariada. Por esta razón, el análisis se realizó con la muestra inicial $(n=560)$. Específicamente, se llevó a cabo el análisis factorial confirmatorio con la estructura factorial de cinco factores encontrada en el análisis factorial exploratorio.

El proceso del análisis fue el siguiente. En principio, se examinó la normalidad multivariada de dos formas: primero se analizó el coeficiente de Mardia mediante el programa EQS, versión 6.1, con el cual se encontró un coeficiente normalizado, cuyo valor fue de 97.7592 — según Ullman (2006), un coeficiente normalizado superior a 3.00 indica la no normalidad, por lo que se estima que no hay normalidad multivariada-; $y$ con el fin de precisar este supuesto de normalidad, se realizó la prueba de Shapiro-Wilk generalizada — que determina

Tabla 3.

Índices de ajuste para el modelo de cinco factores sin los items 2, 6, 23 y 24

\begin{tabular}{cccccccc}
\hline Índices & KMO & $\chi^{2}$ & $d f$ & $\chi^{2} / d f$ & $p$ & RMSEA [IC 90 \%] & TLI \\
\hline Modelo de cinco factores & 0.892 & 152.666 & 100 & 1.52 & $<.001$ & $0.049[0.031,0.06]$ & 0.952 \\
\hline
\end{tabular}


Tabla 4.

Estructura factorial exploratoria con un modelo de cinco factores

$\begin{array}{lccccc}\text { Ítem } & \text { Factor } 1 & \text { Factor } 2 & \text { Factor } 3 & \text { Factor } 4 & \text { Factor } 5 \\ \text { Relaciones } & \begin{array}{c}\text { Cambio } \\ \text { interpersonales }\end{array} & \text { Toknecesario } & \text { Tokenismo } & \begin{array}{c}\text { Falta de } \\ \text { conocimiento }\end{array} & \begin{array}{c}\text { Metas y a } \\ \text { conflictivas }\end{array}\end{array} h^{2}$

1. No hay razones por las cuales debería hacer este cambio porque me siento confiado en que las innovaciones

.545

.328

tecnológicas resolverán los problemas ambientales.

3. Estos problemas están tan lejos en el futuro, que no hay necesidad de actuar.

.853

4. Cambios como este no son realmente necesarios para mí porque es probable que las condiciones ambientales se mantengan bien en mi área.

5. No hay necesidad urgente de cambio porque la naturaleza tiene gran resiliencia y nuestras acciones son triviales.

7. Me preocupa que este cambio tome gran parte de mi tiempo.

8. No puedo cambiar porque he invertido en mi estilo de vida actual.

9. Estos temas son importantes para mí, pero es muy difícil cambiar mis hábitos.

10. No he cambiado porque temo que esto no funcionaría.

11. Hacer este cambio sería criticado por los que me rodean.

12. Estaría defraudando a ciertas personas si hiciera este cambio.

13. Me preocupa que mis amigos me desaprueben si hago este cambio.

14. Si realizara el cambio necesario, probablemente me sentiría avergonzado cuando otros se dieran cuenta de lo que estaba haciendo.

15. Hay tanta información ahí fuera que estoy confundido sobre cómo hacer este cambio.

16. No entiendo lo suficiente de los detalles sobre cómo hacer este cambio.

17. Me gustaría cambiar, pero no estoy seguro de dónde empezar.

18. Los esfuerzos en pro del ambiente en los que estoy actualmente involucrado hacen que cambios adicionales sean innecesarios.

19. Ya he hecho sacrificios para resolver problemas ambientales, así que no hay necesidad de hacer más.

20. Anteriormente he hecho un esfuerzo importante en este sentido, por lo que no es necesario que haga más cambios.

21. Mis acciones ambientales ya hacen suficiente diferencia.

22. No es justo para mí cambiar cuando en realidad es la industria la que está causando la mayoría de los problemas ambientales.

Nota Todos los pesos factoriales son $>.32 . h^{2}=$ comunalidad. 
Tabla 5.

Índices de ajuste robustos para el modelo factorial de cinco factores

\begin{tabular}{lcccccccc}
\hline \multicolumn{1}{c}{ Modelo } & Satorra-Bentler $\chi^{2}$ & $d f$ & $\chi^{2} / d f$ & $\begin{array}{c}\text { RMSEA robusto } \\
{[\text { CI 90 \% }]}\end{array}$ & SRMR robusto & CFI robusto & $\begin{array}{c}\text { NNFI } \\
\text { robusto }\end{array}$ & IFI robusto \\
\hline $\begin{array}{l}\text { Estructura de cinco } \\
\text { factores }\end{array}$ & 284.0553 & 155 & 1.83 & $\begin{array}{c}0.039 \\
{[0.031,0.046]}\end{array}$ & 0.057 & 0.939 & 0.925 & 0.940 \\
\hline
\end{tabular}

como hipótesis nula una distribución multivariada, pero, si el valor $p$ es inferior al valor alfa, esta se rechaza (VillasenorAlva \& Estrada, 2009) - , con lo cual se obtuvo un valor $p<.001$, de manera que fue posible rechazar la hipótesis nula y comprobar la ausencia de normalidad multivariada de los datos. Por ende, se puede afirmar que la distribución no presenta normalidad multivariada.

Pese a la falta de normalidad, debido a que Martínez et al. (2014) recomiendan asumir el supuesto de normalidad multivariada, y con el fin de evitar la afectación de las estimaciones por la ausencia de normalidad, se utilizó un método robusto (Finney \& DiStefano, 2006). De esta manera, se llevó a cabo el análisis —en EQS, versión 6.1 — con el método de estimación de máxima verosimilitud-robusto, mediante el cual se obtuvieron índices robustos a partir del estadístico $\chi^{2}$ de Satorra-Bentler. Esta prueba corrige los posibles errores causados por la ausencia de normalidad, y con esto los índices obtenidos resultan ser adecuados (Sandin et al., 2007).

Continuando con el análisis, los índices examinados para determinar el ajuste del modelo fueron la relación entre el $\chi^{2}$ de Satorra-Bentler y los grados de libertad $\left(\chi^{2} / d f\right)$ - en la cual se sugiere obtener un valor $\leq 5$-, el RMSEA - cuyo valor esperado es $<0.08$ - el SRMR —en el cual se espera un valor $\leq 0.08-\mathrm{y}$, los índices CFI, IFI, y NNFI —en los cuales se sugiere obtener un valor $\geq 0.90$ - (Martínez et al., 2014; Ruiz et al., 2010; Sandin et al., 2007). Como se observa en la Tabla 5, los índices de bondad de ajuste indican un ajuste muy satisfactorio del modelo de cinco factores.

Por otra parte, como se encuentra en la Tabla 6, la estimación de los parámetros robustos fue superior a 0.45 para cada uno de los ítems, lo cual indica la importancia que tiene cada factor en la identificación del reactivo (Rigo \& Donolo, 2018); y, como se observa en la Tabla 7, las covarianzas entre factores están dentro del intervalo de confianza, no se encontraron varianzas negativas, y ningún parámetro excedió el valor de 1 (Herrero, 2010). En las Tablas 6 y 7, el valor del error fue inferior a la estimación, $\mathrm{y}$ todas las estimaciones fueron significativas $(p<.001)$.
Tabla 6.

Estimación de parámetros estandarizados con métodos robustos

\begin{tabular}{lccccc}
\hline \multicolumn{1}{c}{ Factor } & \multicolumn{5}{c}{ Indicador Estimación Error Valor Z } \\
\hline Relaciones & DIPB 11 & 0.635 & 0.096 & 6.585 & $<.001$ \\
interperso- & DIPB 12 & 0.680 & 0.111 & 6.138 & $<.001$ \\
nales & DIPB 13 & 0.691 & 0.117 & 5.913 & $<.001$ \\
& DIPB 14 & 0.656 & 0.111 & 5.900 & $<.001$ \\
& & & & & \\
Cambio & DIPB 1 & 0.578 & 0.070 & 8.283 & $<.001$ \\
innecesario & DIPB 3 & 0.652 & 0.086 & 7.552 & $<.001$ \\
& DIPB 4 & 0.744 & 0.080 & 9.280 & $<.001$ \\
& DIPB 5 & 0.704 & 0.110 & 6.423 & $<.001$ \\
& & & & & \\
& DIPB 18 & 0.585 & 0.092 & 6.328 & $<.001$ \\
Tokenismo & DIPB 19 & 0.717 & 0.110 & 6.510 & $<.001$ \\
& DIPB 20 & 0.819 & 0.126 & 6.498 & $<.001$ \\
& DIPB 21 & 0.672 & 0.110 & 6.135 & $<.001$ \\
& DIPB 22 & 0.605 & 0.097 & 6.244 & $<.001$ \\
Falta de & DIPB 15 & 0.732 & 0.035 & 21.078 & $<.001$ \\
conocimiento & DIPB 16 & 0.869 & 0.035 & 24.879 & $<.001$ \\
& DIPB 17 & 0.707 & 0.047 & 14.940 & $<.001$ \\
Metas y & & & & & \\
aspiraciones & DIPB 7 & 0.706 & 0.090 & 7.872 & $<.001$ \\
conflictivas & DIPB 8 & 0.681 & 0.098 & 6.950 & $<.001$ \\
& DIPB 9 & 0.488 & 0.062 & 7.916 & $<.001$ \\
& DIPB 10 & 0.630 & 0.084 & 7.487 & $<.001$ \\
\hline
\end{tabular}

Adicional a esto, en la Tabla 7 se pueden observar covarianzas fuertes - de 0.732 a 0.787 - entre los factores relaciones interpersonales, cambio innecesario, tokenismo y metas y aspiraciones conflictivas; así como covarianzas bajas — de 0.091 a 0.397 - entre el factor falta de conocimiento y los demás factores. Las correlaciones bajas podrían deberse a que el factor de falta de conocimiento se refiere en su totalidad a una cognición limitada o a un desconocimiento acerca de cómo cambiar el comportamiento (Lacroix et al., 2019), lo cual puede diferir un poco frente a los demás factores.

Finalmente, en la Figura 1 se presenta la estructura factorial mediante un path diagram con sus respectivas correlaciones y estimaciones de parámetros estandarizados. La escala aplicable se encuentra en el Apéndice A. 
Tabla 7.

Covarianzas entre factores, valores robustos

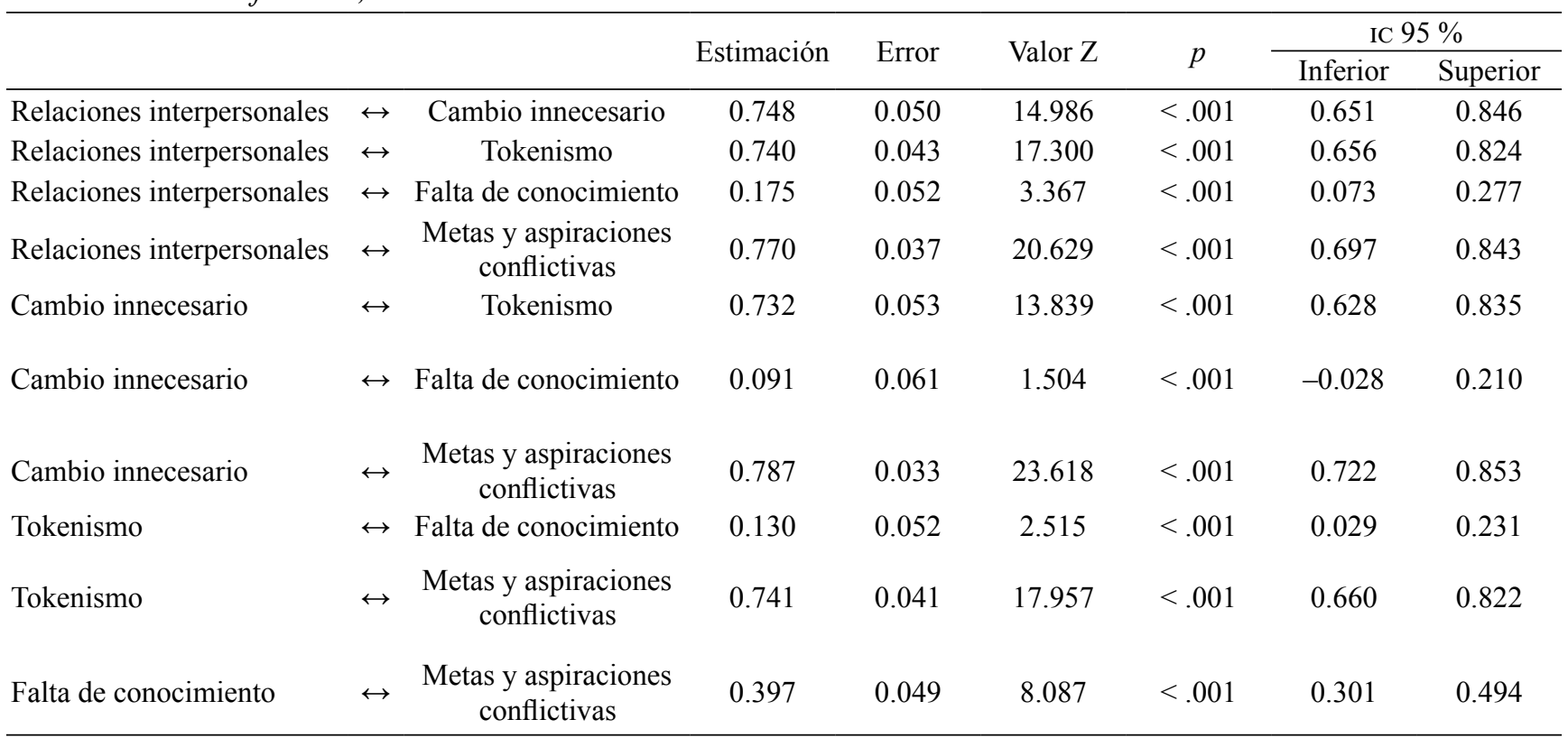

Figura 1.

Modelo de cinco factores con estimaciones de parámetros estandarizados

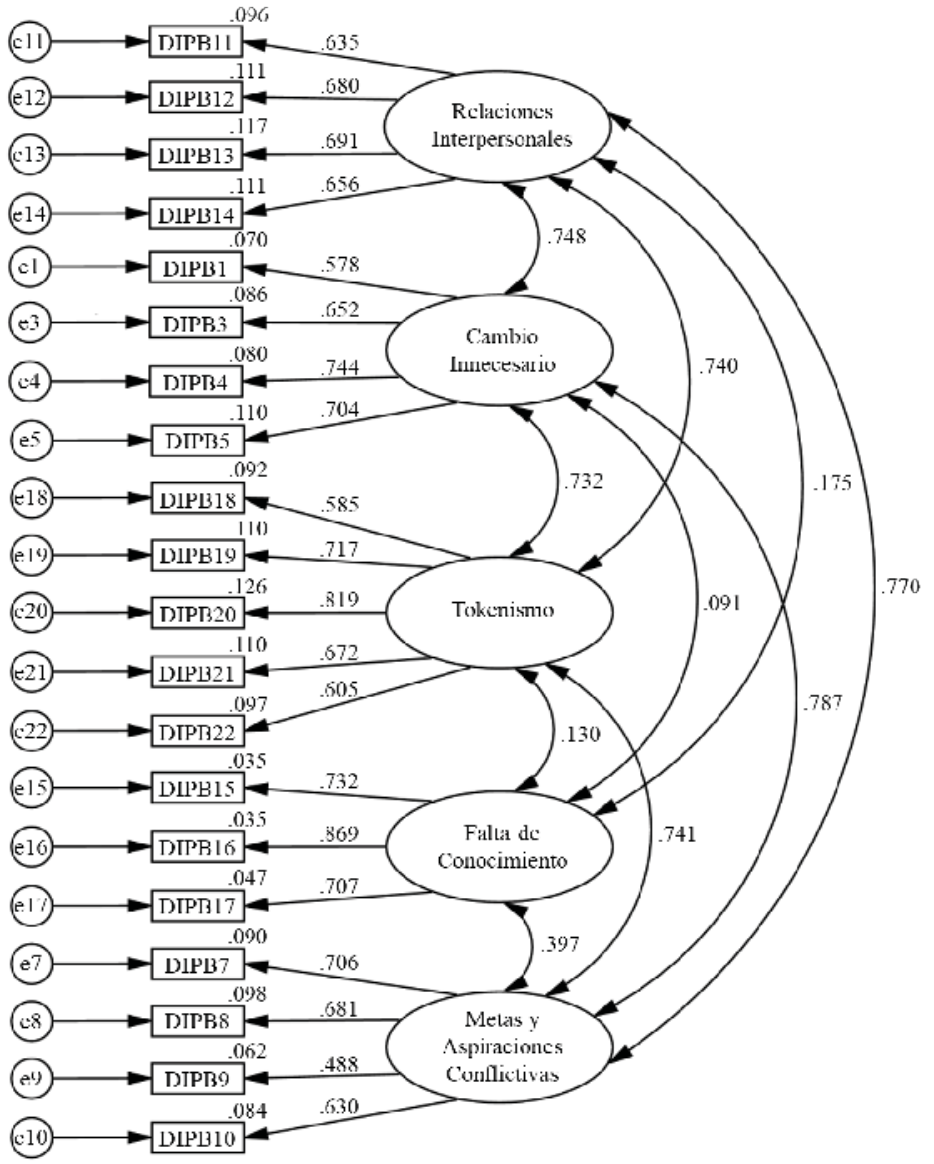


Análisis de reactivos y fiabilidad del instrumento

$\mathrm{El}$ análisis de reactivos y el análisis de fiabilidad del instrumento fueron realizados con una muestra de 560 participantes - 270 hombres (48.21\%) y 290 mujeres (51.79\%)-. Específicamente, se llevó a cabo el análisis de fiabilidad para cada factor y para el instrumento en su totalidad, y para ello se tuvieron en cuenta como indicadores el omega de McDonald, el alfa de Cronbach y el coeficiente de Guttman. Como se observa en la Tabla 8, los coeficientes de fiabilidad muestran, en general, valores superiores a .70 para cada factor, lo cual da cuenta de que el instrumento cuanta con una fiabilidad alta en cada uno de ellos (Barbero, 2010; Oviedo \& Campo-Arias, 2005); al igual que en la escala total ( $\omega$ de McDonald $=.906$, $\alpha$ de Cronbach $=.902$, y $\lambda 6$ de Guttman $=.924)$, lo cual demuestra que el instrumento completo también cuenta con una fiabilidad bastante alta (Barbero, 2010).

Por otra parte, los resultados del análisis de reactivos a través de la correlación ítem-total se muestran en la Tabla 9. En ella, se puede observar que todas las correlaciones entre reactivos son mayores a 0.30 — este valor se considera como
Tabla 8.

Coeficientes de fiabilidad para cada factor

\begin{tabular}{lccc}
\hline \multicolumn{1}{c}{ Factor } & $\begin{array}{c}\omega \text { de } \\
\text { McDonald }\end{array}$ & $\begin{array}{c}\alpha \text { de } \\
\text { Cronbach }\end{array}$ & $\begin{array}{c}\lambda 6 \text { de } \\
\text { Guttman }\end{array}$ \\
\hline Relaciones & .881 & .879 & .852 \\
interpersonales & .790 & .780 & .742 \\
Cambio innecesario & .778 & .770 & .744 \\
Tokenismo & .817 & .812 & .751 \\
Falta de conocimiento & .750 & .742 & .698 \\
Metas y aspiraciones & & .902 & .924 \\
conflictivas & & & \\
Escala total & & &
\end{tabular}

el criterio de aceptación para cada reactivo (De los SantosRoig \& Pérez-Meléndez, 2014)—, y que la mayoría de los ítems se ajustan, ya que, al ser eliminados, la fiabilidad del factor disminuye. No obstante, aunque los ítems 1, 9 y 22 no cumplen este criterio, se considera adecuado dejarlos como parte del instrumento, debido a que la fiabilidad no se afecta en gran medida, porque la diferencia se presenta en el tercer decimal, y porque los reactivos superan el valor de 0.30 en la correlación.

Tabla 9.

Análisis de los reactivos, correlación ítem-total

\begin{tabular}{|c|c|c|}
\hline Factores e ítems & $\begin{array}{l}\text { Correlación total de } \\
\text { elementos corregida }\end{array}$ & $\begin{array}{l}\text { Alfa de Cronbach } \\
\text { si el elemento se } \\
\text { ha suprimido }\end{array}$ \\
\hline \multicolumn{3}{|l|}{ Factor 1: Relaciones interpersonales $(\alpha=.879)$} \\
\hline 11. Hacer este cambio sería criticado por los que me rodean. & .662 & .875 \\
\hline 12. Estaría defraudando a ciertas personas si hiciera este cambio. & .760 & .836 \\
\hline 13. Me preocupa que mis amigos me desaprueben si hago este cambio. & .789 & .824 \\
\hline $\begin{array}{l}\text { 14. Si realizara el cambio necesario, probablemente me sentiría avergonzado } \\
\text { cuando otros se dieran cuenta de lo que estaba haciendo. }\end{array}$ & .738 & .844 \\
\hline \multicolumn{3}{|l|}{ Factor 2: Cambio innecesario $(\alpha=.780)$} \\
\hline $\begin{array}{l}\text { 1. No hay razones por las cuela debería hacer este cambio porque me siento } \\
\text { confiado en que las innovaciones tecnológicas resolverán los problemas } \\
\text { ambientales. }\end{array}$ & .414 & .810 \\
\hline 3. Estos problemas están tan lejos en el futuro, que no hay necesidad de actuar. & .621 & 699 \\
\hline $\begin{array}{l}\text { 4. Cambios como este no son realmente necesarios para mí porque es probable } \\
\text { que las condiciones ambientales se mantengan bien en mi área. }\end{array}$ & .633 & .695 \\
\hline $\begin{array}{l}\text { 5. No hay necesidad urgente de cambio porque la naturaleza tiene gran resilien- } \\
\text { cia y nuestras acciones son triviales. }\end{array}$ & .641 & .688 \\
\hline \multicolumn{3}{|l|}{ Factor 3: Tokenismo $(\alpha=.770)$} \\
\hline $\begin{array}{l}\text { 18. Los esfuerzos en pro del ambiente en los que estoy actualmente involucrado } \\
\text { hacen que cambios adicionales sean innecesarios. }\end{array}$ & .458 & .753 \\
\hline $\begin{array}{l}\text { 19. Ya he hecho sacrificios para resolver problemas ambientales, así que no hay } \\
\text { necesidad de hacer más. }\end{array}$ & .604 & .701 \\
\hline $\begin{array}{l}\text { 20. Anteriormente he hecho un esfuerzo importante en este sentido, por lo que } \\
\text { no es necesario que haga más cambios. }\end{array}$ & .630 & .690 \\
\hline 21. Mis acciones ambientales ya hacen suficiente diferencia. & .592 & .709 \\
\hline $\begin{array}{l}\text { 22. No es justo para mí cambiar cuando en realidad es la industria la que está } \\
\text { causando la mayoría de los problemas ambientales. }\end{array}$ & .392 & .777 \\
\hline
\end{tabular}




\begin{tabular}{lll}
\hline Factor 4: Falta de conocimiento $(\alpha=.812)$. & \\
15. Hay tanta información ahí fuera que estoy confundido sobre cómo hacer este & .637 & .767 \\
$\quad$ cambio. & .730 & .671 \\
16. No entiendo lo suficiente de los detalles sobre cómo hacer este cambio. & .620 & .784 \\
17. Me gustaría cambiar, pero no estoy seguro de dónde empezar. & & .652 \\
Factor 5: Metas y aspiraciones conflictivas $(\alpha=.742)$ & .578 & .640 \\
7. Me preocupa que este cambio tome gran parte de mi tiempo. & .603 & .745 \\
8. No puedo cambiar porque he invertido en mi estilo de vida actual. & .422 & .688 \\
9. Estos temas son importantes para mí, pero es muy difícil cambiar mis & .524 & \\
$\quad$ hábitos. & & \\
\hline
\end{tabular}

Finalmente, en la Tabla 10 se observa la comparación entre la estructura del instrumento original y la estructura de la adaptación realizada en el presente estudio — con sus respectivos indicadores del alfa de Cronbach-. En general, se puede observar que ambas estructuras cuentan con cinco factores - aunque el orden de los factores es distinto-; que los ítems que componen la versión de la prueba adaptada tienen la misma distribución que la estructura original; que el instrumento original tuvo coeficientes de fiabilidad más altos en los factores de cambio innecesario y metas y aspiraciones conflictivas; que tanto la versión original como la adaptada tuvieron el mismo coeficiente de fiabilidad en el factor tokenismo; y que en este estudio se obtuvieron coeficientes de fiabilidad más altos en los factores de relaciones interpersonales y de falta de conocimiento.

\section{Análisis de invarianza}

Se realizaron dos estudios de invarianza mediante el método de invarianza de configuración. Este método consiste en contrastar los índices de bondad de ajuste mediante el análisis de la estructura factorial con ambos grupos de estudio por separado, con el fin de establecer si los indicadores se comportan de manera similar, y si el modelo se ajusta en ambos grupos (Abad et al., 2011). Así, primero se evaluó la estructura factorial con dos muestras de tamaño distinto, y luego se realizó el estudio de invarianza por sexo. Para los dos estudios se utilizó un método de máxima verosimilitud-robusto y se examinaron los siguientes índices robustos: la relación entre el $\chi^{2}$ de Satorra-Bentler y los grados de libertad $\left(\chi^{2} / d f\right)$, el RMSEA, el SRMR, el CFI, el IFI y el NNFI, ya comentados.

Tabla 10.

Comparación entre la estructura original del instrumento y la estructura obtenida mediante la adaptación

\begin{tabular}{|c|c|c|c|c|c|c|c|}
\hline \multicolumn{4}{|c|}{$\begin{array}{c}\text { Escala con cinco factores } \\
\text { prueba original }\end{array}$} & \multicolumn{4}{|c|}{$\begin{array}{c}\text { Escala con cinco factores } \\
\text { prueba adaptada (nueva numeración) }\end{array}$} \\
\hline \multicolumn{2}{|c|}{ Componentes } & \multirow{2}{*}{$\frac{\text { Ítems }}{1,2,3,4 \text { y } 5}$} & \multirow{2}{*}{$\frac{\alpha}{0.85}$} & \multicolumn{2}{|r|}{ Componentes } & \multirow{2}{*}{$\frac{\text { Ítems }}{9,10,11 \text { y } 12}$} & \multirow{2}{*}{$\begin{array}{c}\alpha \\
0.879\end{array}$} \\
\hline Factor 1 & Cambio innecesario & & & Factor 1 & $\begin{array}{l}\text { Relaciones } \\
\text { interpersonales }\end{array}$ & & \\
\hline Factor 2 & $\begin{array}{l}\text { Metas y aspiraciones } \\
\text { conflictivas }\end{array}$ & $6,7,8,9$ y 10 & 0.82 & Factor 2 & Cambio innecesario & $1,2,3$ y 4 & 0.780 \\
\hline Factor 3 & $\begin{array}{l}\text { Relaciones } \\
\text { interpersonales }\end{array}$ & $11,12,13$ y 14 & 0.80 & Factor 3 & Tokenismo & $16,17,18,19$ у 20 & 0.770 \\
\hline Factor 4 & $\begin{array}{l}\text { Falta de } \\
\text { conocimiento }\end{array}$ & 15,16 y 17 & 0.73 & Factor 4 & Falta de conocimiento & 13,14 y 15 & 0.812 \\
\hline Factor 5 & Tokenismo & $18,19,20,21$ y 22 & 0.77 & Factor 5 & $\begin{array}{l}\text { Metas y aspiraciones } \\
\text { conflictivas }\end{array}$ & $5,6,7$ y 8 & 0.742 \\
\hline Escala total & & & & & & & 0.902 \\
\hline
\end{tabular}


Primero, se utilizó una muestra de 460 y otra de 560 sujetos, y se obtuvieron los índices robustos para ambos grupos. En la muestra de 460 se encontraron los índices: $\chi^{2}$ de Satorra-Bentler $=303.3019, d f=155, \chi^{2} / d f=1.95$, RMSEA $[\mathrm{IC} 90 \%]=0.046[0.038,0.053], \mathrm{SRMR}=0.061, \mathrm{CFI}=0.925$, $\mathrm{IFI}=0.927 \mathrm{y}$ NNFI $=0.908$. Y en la muestra de 560 , se obtuvieron los índices: $\chi^{2}$ de Satorra-Bentler $=284.0553$, $d f=155, \chi^{2} / d f=1.83$, RMSEA [IC $\left.90 \%\right]=0.039[0.031$, $0.046], \mathrm{SRMR}=0.057, \mathrm{CFI}=0.939, \mathrm{IFI}=0.940 \mathrm{y} \mathrm{NNFI}=0.925$. Con estas puntuaciones, se puede observar que los índices de ajuste son semejantes en ambos grupos, es decir, que la estructura factorial es funcional para ambos grupos.

Posteriormente, se realizó el estudio de invarianza por sexo, en el cual se utilizó una muestra conformada por 270 hombres $(48.21 \%)$ y 290 mujeres $(51.79 \%)$. Para el grupo de mujeres se obtuvieron los índices: $\chi^{2}$ de Satorra-Bentler $=212.0077, d f=155, \chi^{2} / d f=1.36$, RMSEA $[\mathrm{IC} 90 \%]=0.036[0.023,0.047], \mathrm{SRMR}=0.072, \mathrm{CFI}=0.925$, $\mathrm{IFI}=0.929 \mathrm{y} \mathrm{NNFI}=0.909 ; \mathrm{y}$ para el grupo de hombres se obtuvieron los índices: $\chi^{2}$ de Satorra-Bentler $=213.2368$, $d f=155, \chi^{2} / d f=1.37$, RMSEA [IC $\left.90 \%\right]=0.037[0.024$, $0.049], \mathrm{SRMR}=0.055, \mathrm{CFI}=0.951, \mathrm{IFI}=0.952 \mathrm{y} \mathrm{NNFI}=0.940$. Con esto, se determinó que los índices de ajuste se comportaron de forma similar para ambos grupos, de manera que se puede afirmar que la estructura de cinco factores se comporta de forma adecuada para ambos sexos, al ratificar la invarianza del modelo en ambos grupos.

\section{Baremación}

Para la baremación del instrumento se utilizó una muestra inicial de 560 participantes, 270 hombres (48.21\%) y 290 mujeres (51.79\%). En primera instancia, se realizó un análisis de normalidad para examinar si era posible establecer baremos para cada uno de los factores. Para ello, se tuvo en cuenta la prueba de normalidad de Kolmogorov-Smirnov, con la cual se determinó que ninguno de los factores contaba con distribución normal. Posteriormente, se realizó una comparación por género usando la prueba no paramétrica $U$ de Mann Whitney, con la cual no se encontraron diferencias significativas. Así, se decidió realizar la baremación para el total del instrumento.

Inicialmente se había planteado realizar la baremación mediante datos semiagrupados, pero, de esta manera, resultaron demasiadas categorías, tanto así que dificultaban la comprensión del instrumento de medición. Por esta razón, se decidió utilizar una distribución de datos agrupada.

Para ello, se eliminaron 25 datos extremos, con lo cual la muestra quedó conformada por 535 participantes, 252 hombres (47\%) y 283 mujeres (53\%). Después de esto, se obtuvieron las calificaciones para las siete categorías, atendiendo a las opciones de respuesta del instrumento — este procedimiento de agrupación está adecuado desde lo estadístico y desde el punto de vista de la interpretación de la prueba—. Finalmente, se realizó la interpretación de cada una de las categorías con base en la teoría de las barreras psicológicas, denominada en el artículo original como dragones de inacción (Gifford, 2011; Lacroix et al., 2019).

A partir de esto, se determinó el rango centil, la puntuación $Z$ y la puntuación $\mathrm{T}(M=50$ y $D E=10)$ para cada intervalo de las puntuaciones directas. Además, se contemplaron siete categorías en las que puede puntuar una persona: en la primera categoría - llamada "Muy bajo"- se encuentran aquellas personas que puntúan entre 20 y 28 , quienes se considerarían personas con un interés alto por el medio ambiente y que realizan acciones proambientales; en la categoría intermedia - "Promedio"- se encuentran los sujetos que puntúan entre 47 y 55, que son aquellos que a veces pueden realizar acciones proambientales y otras veces no; y en la última categoría - "Muy alto"-, están quienes puntúan entre 74 y 82 , y son sujetos que en general no demuestran preocupación por el medio ambiente y no actúan en pro del ambiente.

\section{Discusión}

En vista de la funcionalidad de la escala Dragons of Inaction Psychological Barriers (DIPB) (Lacroix et al., 2019), el objetivo de la presente investigación fue realizar la adaptación de este instrumento en población colombiana. Para ello, se emplearon las directrices de adaptación de Muñiz et al. (2013).

En principio, se realizó un proceso de back translation, con el cual se obtuvo una traducción de la escala apropiada para el contexto. Luego, se llevó a cabo la validación por jueces, siguiendo las recomendaciones propuestas por Escobar-Pérez y Cuervo-Martínez (2008), y en este proceso, los ítems demostraron tener una adecuada coherencia, 
relevancia y claridad, a la vez que los factores mostraron alta suficiencia. Teniendo esto en cuenta, no se hizo ninguna modificación de la escala y fue posible su aplicación con el fin de realizar los respectivos análisis.

Después de esto, se realizó un análisis factorial exploratorio, en el cual la prueba fue sometida a procedimientos para uno, dos, tres, cuatro, cinco, seis y siete factores, con lo cual se encontró una estructura multifactorial interpretable y satisfactoria de cinco factores, lo que concuerda con la escala original de Lacroix et al. (2019). Además, los reactivos que conforman la versión del instrumento adaptado poseen la misma distribución que la versión original, aunque el orden de los factores es distinto. Para adecuar esta estructura factorial, se excluyeron los ítems 2 y 6 , pues no saturaban en ningún factor, y los ítems 23 y 24 , los cuales formaban su propio factor, y lo recomendable es tener mínimo tres variables en cada factor para poder establecerlo (Raubenheimer, 2004). Los ítems 23 y 24 también fueron eliminados en el estudio original (Lacroix et al., 2019).

Posteriormente, se puso a prueba la estructura factorial encontrada en el análisis factorial exploratorio, y se realizó el análisis factorial confirmatorio, en el cual se examinaron los siguientes índices: la relación entre el $\chi^{2}$ de Satorra-Bentler y los grados de libertad $\left(\chi^{2} / d f\right)$, el RMSEA, el SRMR, el CFI, el IFI y el NNFI. Con los puntajes obtenidos, se comprobó que la estructura factorial de cinco factores es efectiva, ya que muestra índices de bondad de ajuste muy buenos. Adicional a esto, se llevó a cabo el análisis de reactivos, y todas las variables obtuvieron una correlación satisfactoria de acuerdo con lo esperado, es decir, con valores mayores a 0.30 (De los Santos-Roig \& Pérez-Meléndez, 2014).

Por otro lado, en el análisis de fiabilidad se analizaron los siguientes coeficientes: omega de McDonald, alfa de Cronbach y el coeficiente de Guttman. En general, la fiabilidad para cada factor y para la escala total fue considerada alta, es decir, que en todos los casos se obtuvo valores mayores a 0.70 (Barbero, 2010; Oviedo \& Campo-Arias, 2005). Específicamente, los resultados del coeficiente alfa de Cronbach fueron los siguientes: primer factor, relaciones interpersonales, .879; segundo factor, cambio innecesario, .780; tercer factor, tokenismo, .770; cuarto factor, falta de conocimiento, .812; y quinto factor, metas y aspiraciones conflictivas, .742. Asimismo, el instrumento completo cuenta con una fiabilidad alta, dado que el alfa de Cronbach total fue de .902 .
En cuanto a la comparación con el estudio original, se evidenció que la escala original tuvo coeficientes de fiabilidad más altos en los factores cambio innecesario y metas $y$ aspiraciones conflictivas; en cambio, en este estudio los factores relaciones interpersonales y falta de conocimiento tuvieron una fiabilidad mayor. El factor tokenismo tuvo el mismo coeficiente en los dos estudios. Por otra parte, en este estudio la fiabilidad del instrumento fue alta, pero este dato no se pudo comparar con la escala original, ya que en ese trabajo no se reporta dicho coeficiente.

A partir de los análisis realizados la escala quedo conformada por 20 reactivos con una nueva numeración de la siguiente forma: el primer factor, relaciones interpersonales, quedó conformado por los ítems 9, 10, 11 y 12; el segundo factor, cambio innecesario, por los ítems 1, 2, 3 y 4; el tercer factor, tokenismo, por los ítems 16, 17, 18, 19 y 20; el cuarto factor, falta de conocimiento, por los ítems 13, 14 y 15; y el quinto factor, metas y aspiraciones conflictivas, por los ítems 5, 6, 7 y 8. Este modelo de cinco factores explica el $54.6 \%$ de la varianza total, lo cual se considera muy apropiado, puesto que se espera que el modelo explique un mínimo del $40 \%$ de la varianza (Lloret-Segura et al., 2014). La escala final, para su aplicación, se puede encontrar en el Apéndice A.

Por otra parte, los factores del instrumento, según Lacroix et al. (2019), se relacionan con los aspectos descritos a continuación: (a) el factor relaciones interpersonales está ligado a temas de crítica o desaprobación social; (b) cambio innecesario se refiere a la negación de los problemas ambientales, y, con ello, la negación de la necesidad de actuar, por lo cual el cambio de comportamiento se considera innecesario; (c) tokenismo incluye la creencia de que los cambios de comportamiento realizados individualmente son suficientes, y que otras entidades externas deberían facilitar el cambio de comportamiento propio; (d) falta de conocimiento representa la ignorancia en términos de no saber cómo realizar un cambio de comportamiento; y (e) metas $y$ aspiraciones conflictivas incluye objetivos que pueden verse afectados si se trata de cambiar el comportamiento, lo que implica la limitación del tiempo o la dificultad para cambiar hábitos.

Continuando con los análisis, en este estudio se probó la invarianza con dos muestras de tamaño distinto, así como la invarianza por sexo. En ambos estudios de invarianza se examinaron los índices siguientes índices: la relación 
entre el $\chi^{2}$ de Satorra-Bentler y los grados de libertad $\left(\chi^{2} / d f\right)$, el RMSEA, el SRMr, el CFI, el IFI y el NNFI. Los valores de estos índices fueron satisfactorios en los dos casos, lo que confirma que tanto los ítems como los factores significan lo mismo para todos los grupos. Es necesario resaltar este hallazgo, ya que, aunque en el estudio original se estudió la invarianza por dificultad y dominios ambientales, allí no se analizó la invarianza por muestras de distinto tamaño, y tampoco por sexo (Lacroix et al., 2019).

Finalmente, se realizó la baremación para Colombia, para la puntuación total del instrumento. Para ello, se obtuvieron los rangos centiles, las puntuaciones típicas (puntuación T) y las puntuaciones normalizadas (puntuación Z). Además, se determinaron categorías de las puntuaciones con su respectiva interpretación, con el fin de darle un uso apropiado al instrumento y tener la posibilidad de brindar una interpretación de los resultados en futuros estudios.

Teniendo lo anterior en cuenta, una de las ventajas de la presente investigación es que aporta categorías de análisis para facilitar la interpretación del constructo de las barreras psicológicas. No solo se proporciona un índice cuantitativo del factor y de la escala, sino que, además, se aportan los criterios de interpretación para aplicaciones en este contexto, mientras que el estudio original no indica la baremación respectiva.

En conclusión, con el presente estudio se obtuvo una adaptación apropiada del instrumento Dragons of Inaction Psychological Barriers (DIPB), acá denominado Dragones de Inacción Barreras Psicológicas (DIPB), el cual cuenta con adecuada validez y fiabilidad, y con el que es posible medir las barreras psicológicas —denominadas dragones de inacción - en población colombiana, lo que hará posible realizar un primer diagnóstico respecto a este constructo en Colombia.

Cabe resaltar la importancia de este trabajo investigativo, ya que en contextos latinoamericanos el estudio de las barreras psicológicas que dificultan las conductas proambientales es escaso en comparación con otros contextos que cuentan con mayor desarrollo. De esta manera, se da un paso hacia la dirección correcta para tener un insumo que permita estudiar estas barreras en el contexto colombiano; además, la traducción al español del instrumento puede facilitar el estudio de su validez en diferentes regiones del contexto hispanohablante.

Asimismo, a futuro, este instrumento podría ser utilizado como una medida diagnóstica para evaluar las barreras psicológicas de la población colombiana, con lo cual será posible diseñar programas de intervención, programas educativos o políticas públicas que se centren en la reducción de las barreras psicológicas que impiden la conducta proambiental, de tal forma que la población pueda superar estos obstáculos y se vea un incremento en las acciones proambientales.

Ahora bien, dentro de las limitaciones del estudio se encuentra el tema de la generalización de los resultados, ya que, si bien la muestra se considera de una amplitud adecuada, no tuvo en cuenta varias regiones de Colombia, $\mathrm{y}$, al ser un país tan diverso, estas barreras podrían variar dependiendo del lugar de la aplicación. Es por ello que para futuras investigaciones se sugiere obtener una muestra con mayor amplitud y realizar estudios de invarianza con otras regiones de Colombia. Por último, con la versión adaptada del instrumento, se sugiere realizar investigaciones en Colombia teniendo en cuenta distintos dominios ambientales.

\section{Referencias}

Abad, F., Olea, J., Ponsoda, V., \& García, C. (2011). Medición en ciencias sociales y de la salud. Editorial Síntesis.

Ato, M., López-García, J. J., \& Benavente, A. (2013). Aclassification system for research designs in psychology. Annals of Psychology, 29(3), 1038-1059. https://doi.org/10.60 18/analesps.29.3.178511

Barbero, M. I. (2010). Psicometría: Teoría, formulario y problemas resueltos. Sanz y Torres.

Blake, J. (1999). Overcoming the 'value-action gap' in environmental policy: Tensions between national policy and local experience. Local Environment, 4(3), 257-278. https://doi.org/10.1080/13549839908725599

Carrus, G., Passafaro, P., \& Bonnes, M. (2008). Emotions, habits, and rational choices in ecological behaviours: The case of recycling and use of public transportation. Journal of Environmental Psychology, 28(1), 51-62. https://doi.org/10.1016/j.jenvp.2007.09.003 
Chen, A., \& Gifford, R. (2015). "I wanted to cooperate, but...": Justifying Suboptimal Cooperation in a Commons Dilemma. Canadian Journal of Behavioural Science, 47(4), 282-291. https://doi.org/10.1037/ $\operatorname{cbs} 0000021$

Cheung, G. W., \& Rensvold, R. B. (2009). Evaluating goodness-of-fit indexes for testing measurement invariance. Structural Equation Modeling: A Multidisciplinary Journal, 9(2), 233-255. https://doi.org/10.1207/ S15328007SEM0902_5

Costello, A. B., \& Osborne, J. W. (2005). Best Practices in exploratory factor analysis: Four recommendations for getting the most from your analysis. Practical Assessment, Research and Evaluation, 10(7), 173-178. https://doi.org/10.7275/jyj1-4868

DeVellis, R. F. (1991). Scale development: Theory and applications (3. ${ }^{\mathrm{a}}$ ed.). Sage Publications.

De los Santos-Roig, M., \& Pérez-Meléndez, C. (2014). Items analysis and reliability evidences of ERCE scale. Annals of Psychology, 30(2), 438-449. https://doi.org/10.6018/ analesps.30.2.164021

Díaz-Marín, J. S., \& Geiger, S. (2019). Comportamiento Proambiental: actitudes y valores en una muestra poblacional colombiana. Revista Iberoamericana de Psicología, 12(1), 31-40. https://reviberopsicologia.ibe ro.edu.co/article/view/rip.12103

Dietz, T., Dan, A., \& Shwom, R. (2009). Support for climate change policy: Social psychological and social structural influences. Rural Sociology, 72(2), 185-214. https://doi. org/10.1526/003601107781170026

Escobar-Pérez, J., \& Cuervo-Martínez, A. (2008). Validez de contenido y juicio de expertos: Una aproximación a su utilización. Avances en Medición, 6(1), 27-36. https:// www.researchgate.net/publication/302438451_Validez_ de_contenido_y_juicio_de_expertos_Una_aproxima cion_a_su_utilizacion
Finney, S., \& DiStefano, C. (2006). Non-normal and Categorical data in structural equation modeling. En G. Hancock y R. Mueller (Eds.), Structural equation modeling: a second course (pp. 269-314). Information Age Publishing.

Geiger, N., Middlewood, B., \& Swim, J. (2017). Psychological, social, and cultural barriers to communicating about climate change. Oxford Research Encyclopedia of Climate Science. https://doi. org/10.1093/acrefore/9780190228620.013.377

Gifford, R. (2011). The Dragons of Inaction: Psychological barriers that limit climate change mitigation and adaptation. American Psychologist, 66(4), 290-302. https://doi. org/10.1037/a0023566

Gifford, R., \& Chen, A. (2017). Why aren't we taking action? Psychological barriers to climate-positive food choices. Climatic Change, 140(2), 165-178. https://doi. org/10.1007/s10584-016-1830-y

Herrera-Mendoza, K., Acuña, M., Ramírez, M., \& De la Hoz, M. (2016). Actitud y conducta pro-ecológica de jóvenes universitarios. Opción, 32(13), 456-477. https:// produccioncientificaluz.org/index.php/opcion/article/ view/21609/21413

Herrero, J. (2010). El análisis Factorial Confirmatorio en el estudio de la Estructura y Estabilidad de los Instrumentos de Evaluación: Un ejemplo con el Cuestionario de Autoestima CA-14. Psychological Intervention, 19(3), 289-300. https://doi.org/10.5093/in2010v19n3a9

Intergovernmental Panel on Climate Change [IPCC]. (2021). Climate Change 2021: The Physical Science Basis. Contribution of Working Group I to the Sixth Assessment Report of the Intergovernmental Panel on Climate Change. Cambridge University Press. In Press.

Kollmuss, A., \& Agyeman, J. (2002). Mind the gap: Why do people act environmentally and what are the barriers to pro-environmental behavior? Environmental Education Research, 8(3), 239-260. https://doi. org/10.1080/13504620220145401 
Lacroix, K., \& Gifford, R. (2018). Psychological barriers to energy conservation behavior: The role of worldviews and climate change risk perception. Environment and Behavior, 50(7), 749-780. https://doi. org/10.1177/0013916517715296

Lacroix, K., Gifford, R., \& Chen, A. (2019). Developing and validating the Dragons of Inaction Psychological Barriers (DIPB) scale. Journal of Environmental Psychology, 63, 9-18. https://doi.org/10.1016/j.jenvp.2019.03.001

Leiserowitz, A. (2006). Climate change risk perception and policy preferences: The role of affect, imagery, and values. Climatic Change, 77, 45-72. https://doi. org/10.1007/s10584-006-9059-9

Ley 1090 de 2006. Por la cual se reglamenta el ejercicio de la profesión de Psicología, se dicta el Código Deontológico y Bioético y otras disposiciones. Diario oficial N. 46383 de 6 de septiembre de 2006. Congreso de la República de Colombia. https://www.fun cionpublica.gov.co/eva/gestornormativo/norma_pdf. php?i=66205

Lloret-Segura. S., Ferreres-Traver, A., Hernández-Baeza, A., \& Tomás-Marco, I. (2014). Exploratory Item Factor Analysis: A practical guide revised and up-dated. Annals of Psychology, 30(3), 1151-1169. https://doi.org/10.6018/ analesps.30.3.199361

Lorenzoni, I., Nicholson-Cole, S., \& Whitmarsh, L. (2007). Barriers Perceived to Engaging with Climate Change Among the UK Public and Their Policy Implications. Global Environmental Change, 17(3/4), 445-459. https://doi.org/10.1016/j.gloenvcha.2007.01.004

Martínez, M. R., Hernández, M. J., \& Hernández, M. V. (2014). Psicometría. Alianza Editorial.

Milfont, T. L., \& Fischer, R. (2010). Testing measurement invariance across groups: Applications in cross-cultural research. International Journal of Psychological Research, 3(1), 111-130. https://doi.org/10.21500/20112084.857
Muñiz, J., Elousa, P., \& Hambleton, R. (2013). Directrices para la traducción y adaptación de los tests: segunda edición. Psicothema, 25(2), 151-157. https://doi. org/10.7334/psicothema2013.24

Otzen, T., \& Manterola, C. (2017). Técnicas de Muestreo sobre una Población a Estudio. International Journal of Morphology, 35(1), 227-232. https://doi.org/10.4067/ S0717-95022017000100037

Oviedo, H., \& Campo-Arias, A. (2005). Aproximación al uso del coeficiente alfa de Cronbach. Revista Colombiana de Psiquiatría, 34(4), 572-580. https://www.redalyc.org/ar ticulo.oa?id=80634409

Páramo, P. (2017). Reglas proambientales: una alternativa para disminuir la brecha entre el decir-hacer en la educación ambiental. Suma Psicológica, 24(1), 42-58. https:// doi.org/10.1016/j.sumpsi.2016.11.001

Páramo, P., Sandoval-Escobar, M., Jakovcevic, A., Ferreiro, J., Mustaca, A., Jengich, A., Brenes, J., Ortega-Andeane, P., Vivas, F., Moros, O., Méndez, C., Pasquali, C., Aparecida, M., Anicama, J., Castillo, B., Denegri, M., \& Urzúa, A. (2015). Assessment of environmental quality, degree of optimism, and the assignment of responsibility regarding the state of the environment in Latin America. Universitas Psychologica, 14(2), 605-618. https://doi. org/10.11144/Javeriana.upsy14-2.aeqd

Preuss, S. (1991). Umweltkatastrophe Mensch. Ueber unsere Grenzen und Moeglichkeiten, oekologisch bewusst zu handeln. Roland Asanger Verlag.

Raubenheimer, J. (2004). An item selection procedure to maximise scale reliability and validity. SA Journal of Industrial Psychology, 30(4), 59-64. https://doi. org/10.4102/sajip.v30i4.168

Resolución 8430 de 1993. Por la cual se establecen las normas científicas, técnicas y administrativas para la investigación en salud. 4 de octubre de 1993. Ministerio de Salud, Bogotá, Colombia. https://www.minsalud. gov.co/sites/rid/Lists/BibliotecaDigital/RIDE/DE/DIJ/ RESOLUCION-8430-DE-1993.PDF 
Rigo, D., \& Donolo, D. (2018). Modelos de ecuaciones estructurales usos en investigación psicológica y educativa. Revista Interamericana de Psicología, 52(3), 345-357. https://journal.sipsych.org/index.php/IJP/article/view/388

Ruiz, M., Pardo, A, \& San Martín, R. (2010). Modelos de ecuaciones estructurales. Papeles del Psicólogo, 31(1), 34-45. http://www.papelesdelpsicologo.es/pdf/1794.pdf

Sandin, B., Valiente, R. M., Chorot, P., \& Santed, M. A. (2007). ASI-3: Nueva escala para la evaluación de la sensibilidad a la ansiedad. Revista de Psicopatología y Psicología Clínica, 12(2), 91-104. https://doi. org/10.5944/rppc.vol.12.num.2.2007.4036

Sandoval-Escobar, M., Páramo, P., Orejuela, J., González, I., Cortés, O., Herrera, K., Garzón, C., \& Erazo, C. (2019). Paradojas del comportamiento proambiental de los estudiantes universitarios en diferentes disciplinas académicas. Interdisciplinaria: Revista de Psicología y Ciencias Afines, 36(2), 165-184. https://doi.org/10.16888/ interd.2019.36.2.11

Schultz, P. W., \& Kaiser, F. G. (2012). Promoting pro-environmental behavior. En S. Clayton (ed.), The Oxford handbook of environmental and conservation psychology (pp. 556-580). Oxford University Press.

Stern, P. (2000). New environmental theories: Toward a coherent theory of environmentally significant behavior. Journal of Social Issues, 56(3), 407-424. https://doi. org/10.1111/0022-4537.00175

Stevens, J. (2002). Applied Multivariate statistics for the social sciences. Lawrence Erlbaum.

Stoll-Kleemann, S., O’Riordan, T., \& Jaeger, C. C. (2001). The psychology of denial concerning climate mitigation measures: Evidence from Swiss focus groups. Global Environmental Change, 11(2), 107-117. https://doi. org/10.1016/S0959-3780(00)00061-3
Swim, J., Howard, G., Clayton, S., Reser, J. P., Doherty, T. J., Stern, P. C., Gifford, R., \& Weber, E. U. (2009). Psychology and global climate change: Addressing a multi-faceted phenomenon and set of challenges. American Psychological Association. http://www.apa. org/science/about/publications/climate-change.aspx

Ullman, J. B. (2006). Structural equation modeling. En B. G. Tabachnick \& L. S. Fidell (eds.), Using multivariate stadistics (5. ${ }^{\mathrm{a}}$ ed.) (pp. 653-711). Allyn \& Bacon.

United Nations Environment Programme [UNEP] (2016). Resumen de las evaluaciones regionales GEO-6. https://wedocs.unep.org/bitstream/han dle/20.500.11822/7688/-Resumen_de_las_evalua ciones_regionales_del_sexto_informe_sobre_las_pers pectivaas_del_medio_ambiente_mundial_GEO-6 Resultados_principales_y_mensajes_.pdf? sequence $=3 \&$ isAllowed $=$

Vandenberg, R. J., \& Lance, C. E. (2000). A review and synthesis of the measurement invariance literature: Suggestions, practices, and recommendations for organizational research. Organizational Research Methods, 3(1), 4-70. https://doi.org/10.1177/109442810031002

Villasenor-Alva, J., \& Estrada, E. G. (2009). A Generalization of Shapiro-Wilk's Test for Multivariate Normality. Communications in Statistics-Theory and Methods, 38(11), 1870-1883.https://doi.org/10.1080/03610920802 474465

Wegener, D. T., \& Fabrigar, L. R. (2008). Constructing and evaluating quantitative measures for social psychological research: Conceptual challenges and methodological solutions. The SAGE handbook of methods in social psychology (pp. 145-172). SAGE Publications. http://dx. doi.org/10.4135/9781412976190.n7 


\section{Apéndice A.}

\section{Escala Dragones de Inacción Barreras Psicológicas (DIPB)}

A continuación, se presentan 20 oraciones las cuales hacen referencia a motivos que impiden o dificultan un cambio de comportamiento para realizar acciones proambientales. Por favor, indique en qué medida está MUY EN DESACUERDO (1) o MUY DE ACUERDO (7) en que cada razón es verdadera para usted, puede elegir cualquiera de las 7 opciones.

1. No hay razones por las cuales debería hacer este cambio porque me siento confiado en que las innovaciones tecnológicas resolverán los problemas ambientales.

2. Estos problemas están tan lejos en el futuro, que no hay necesidad de actuar.

3. Cambios como este no son realmente necesarios para mí porque es probable que las condiciones ambientales se mantengan bien en mi área.

4. No hay necesidad urgente de cambio porque la naturaleza tiene gran resiliencia y nuestras acciones son triviales.

5. Me preocupa que este cambio tome gran parte de mi tiempo.

6. No puedo cambiar porque he invertido en mi estilo de vida actual.

7. Estos temas son importantes para mí, pero es muy difícil cambiar mis hábitos.

8. No he cambiado porque temo que esto no funcionaría.

9. Hacer este cambio sería criticado por los que me rodean.

10. Estaría defraudando a ciertas personas si hiciera este cambio.

11. Me preocupa que mis amigos me desaprueben si hago este cambio.

12. Si realizara el cambio necesario, probablemente me sentiría avergonzado cuando otros se dieran cuenta de lo que estaba haciendo

13. Hay tanta información ahí fuera que estoy confundido sobre cómo hacer este cambio.

14. No entiendo lo suficiente de los detalles sobre cómo hacer este cambio.

15. Me gustaría cambiar, pero no estoy seguro de dónde empezar.

16. Los esfuerzos en pro del ambiente en los que estoy actualmente involucrado hacen que cambios adicionales sean innecesarios.

17. Ya he hecho sacrificios para resolver problemas ambientales, así que no hay necesidad de hacer más.

18. Anteriormente he hecho un esfuerzo importante en este sentido, por lo que no es necesario que haga más cambios.

19. Mis acciones ambientales ya hacen suficiente diferencia.

20. No es justo para mí cambiar cuando en realidad es la industria la que está causando la mayoría de los problemas ambientales.

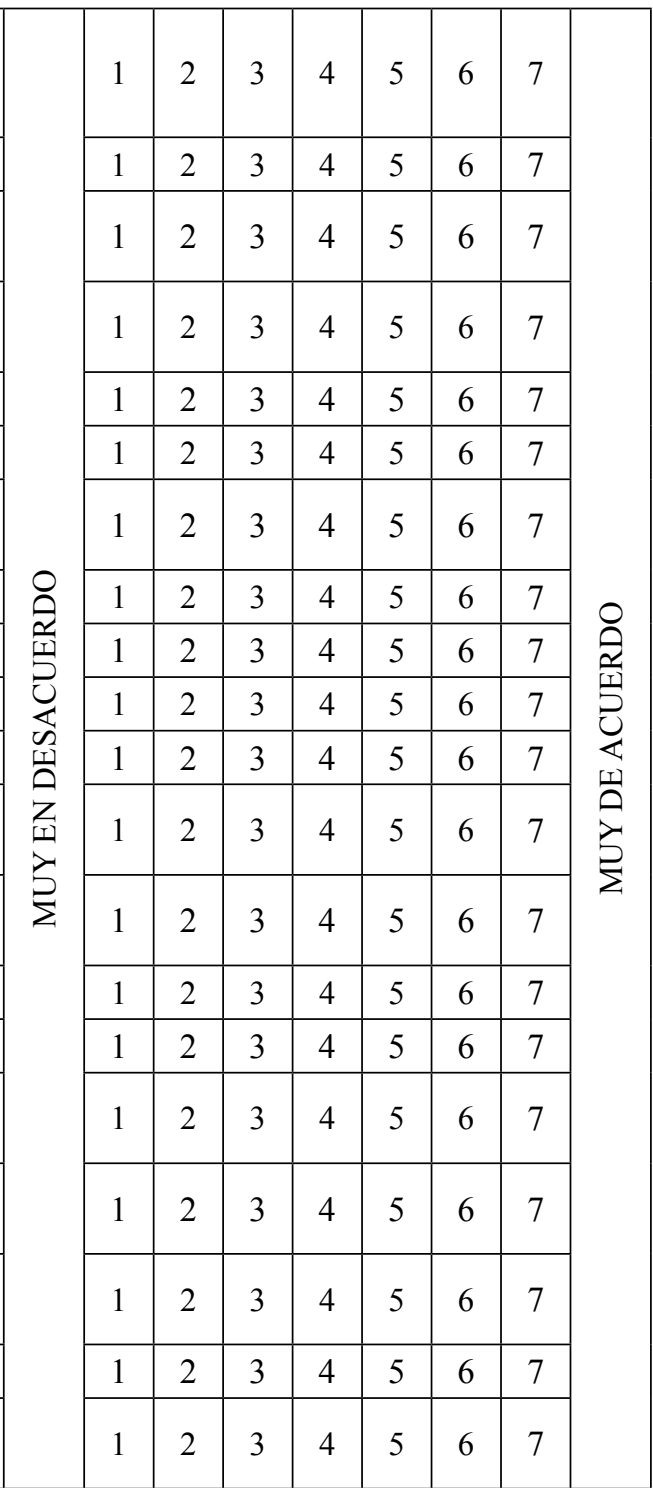

